WUB 96-27

HLRZ 53/96

\title{
Evaluating Sea Quark Contributions to Flavour-Singlet Operators in Lattice QCD
}

\author{
SESAM-Collaboration: \\ N. Eicker ${ }^{\mathrm{a}}$, U. Glässner ${ }^{\mathrm{b}}$, S. Güsken ${ }^{\mathrm{b}}$, H. Hoeber ${ }^{\mathrm{a}}$ \\ Th. Lippert ${ }^{\mathrm{a}}$ G. Ritzenhöfer ${ }^{\mathrm{a}}$, K. Schilling ${ }^{\mathrm{a}, \mathrm{b}}$, G. Siegert ${ }^{\mathrm{a}}$ \\ A. Spitz ${ }^{\mathrm{b}}, \mathrm{P}$. Ueberholz ${ }^{\mathrm{b}}$, and J. Viehoff ${ }^{\mathrm{b}}$ \\ ${ }^{a}$ HLRZ c/o KFA Jülich, D-52425 Jülich \\ and DESY, D-22603 Hamburg, Germany, \\ ${ }^{b}$ Physics Department, University of Wuppertal \\ D-42097 Wuppertal, Germany.
}

\begin{abstract}
In a full QCD lattice study with $N_{f}=2$ Wilson fermions, we seek to optimize the signals for the disconnected contributions to the matrix element of flavoursinglet operators between nucleon states, which are indicative for sea quark effects. We demonstrate, in form of a fluctuation analysis to the noisy estimator technique, that - in order to achieve a tolerable signal to noise-ratio in full QCD - it is advantageous to work with a $Z_{2}$-noise source rather than to rely only on gauge invariance to cancel non-gauge-invariant background. In the case of the $\pi \mathrm{N} \sigma$-term, we find that $10 Z_{2}$-noise sources suffice on our sample ( about 150 independent QCD configurations at $\beta=5.6$ on $16^{3} \times 32$ with $\kappa_{\text {sea }}=0.157$, equivalent to $\left.M_{\pi} / M_{\rho}=0.76(1)\right)$, to achieve decent signals and adequate fluctuations, rather than 300 such sources as recently used in quenched simulations.
\end{abstract}




\section{Introduction}

The direct computation of full fledged hadronic matrix elements containing flavoursinglet operators $\mathcal{A}=\bar{q} \Gamma q$, such as the $\pi$-N $\sigma$-term, $\sigma_{N}$, or the singlet axial vector current forward matrix element between nucleon states, has posed serious problems to lattice gauge theory. As a result there is up to now only faint evidence for sea quark effects from full QCD lattice computations of such matrix elements [1].

The practical bottleneck is given by the computation of disconnected diagrams, i.e. ubiquitous insertions of $\mathcal{A}$ into quark loops disconnected from the valence quark graph, to say the nucleon propagator, $P$, in the vacuum background field. Technically, the disconnected insertion $T=\sum_{x}\left[\operatorname{Tr} \Gamma M_{x \rightarrow x}^{-1}\right]$ must be calculated configurationwise, i.e. in correlation with the hadronic propagator. But the cost to compute all quark loops $M_{x \rightarrow x}^{-1}$ individually grows with volume which renders a direct evaluation of $\sum_{x}$ prohibitively expensive[2].

In the framework of quenched QCD, two groups [3, 4, 5], have recently tackled the problem by applying two different variants of the noisy estimator technique proposed some time ago for computing the chiral condensate $\chi[6]$. The trick is to induce the extensive character of the quark-loop insertion by inverting the Dirac operator $M$ on a source extending over the entire (spatial) volume. This estimator technique is biassed in form of non-closed, i.e. bilocal and therefore non-gauge-invariant contributions that need be controlled.

The Kentucky group [3, 4 pioneered the use of a stochastic volume source with $Z_{2^{-}}$ noise (SETZ) to get rid of the 'bilocal crossterms', $x \rightarrow y$. On their small sample of 50 configurations at $\beta=6.0$, they used about 300 such noise sources per configuration, at the expense of a substantial overhead in their computatinal cost.

The authors of Ref. [5], on the other hand, utilize a homogeneous volume source (VST) and appeal to the gauge fluctuations to suppress the non-loop bias contributions via the Elitzur theorem. This approach however adds a substantial amount of noise to the data and may therefore require a very high statistics ensemble average to yield a reasonable signal.

In exploratory quenched applications, where chromofield configurations can be created at low cost, both approaches have led to rather encouraging results on signal quality[]]. But in the context of a full QCD sea-quark computation, computer time prevents us at present from doing very high statistics sampling of gauge fields. So the question is whether effects from disconnected diagrams are accessible at all in small/medium size sampling of full QCD.

This motivates us to look more deeply into the systematics of the stochastic estimator technique (SET) on actual full QCD configurations with Wilson fermions. To be specific, we will proceed by studying the quality (size and fluctuations) of the signal from disconnected contributions to the scalar density. The strategy in applying estimator techniques should be such that - with minimal overhead - both systematic bias (from remaining nonlocal pollution) and fluctuations of the signal should become negligible compared to the statistical accuracy attainable from the size of the field sample.

On the basis of the current statistics from our hybrid Monte Carlo production 8, 9] we will find that it is realistic to exploit the stochastic estimator technique for the study of quark loop effects in full QCD. 


\section{Asymptotic Expectations}

In order to determine the disconnected part of $\sigma_{N}$ one needs to calculate the expectation value $\left\langle P \operatorname{Tr}\left(M^{-1}\right)\right\rangle$, where $P$ denotes the (momentum zero) proton correlation function and $M$ is the fermion matrix.

With VST one calculates $\sum_{i, j} M_{i, j}^{-1}(C)$ on each gauge configuration $C$ by solving

$$
M(C) X=h
$$

where $h$ is a volume source vector with components $h_{i}=1$. The average over the gauge configurations

$$
A=\frac{1}{N_{C}} \sum_{C=1}^{N_{C}} P(C) \sum_{i, j} M_{i, j}^{-1}(C)
$$

can be separated into local and non local contributions

$$
A=\frac{1}{N_{C}} \sum_{C=1}^{N_{C}} P(C) \sum_{i} M_{i, i}^{-1}(C)+\frac{1}{N_{C}} \sum_{C=1}^{N_{C}} P(C) \sum_{i \neq j} M_{i, j}^{-1}(C) .
$$

As the latter are not gauge invariant they vanish (only) in the large $N_{C}$ limit:

$$
\lim _{N_{C} \rightarrow \infty} A=\left\langle P \operatorname{Tr}\left(M^{-1}\right)\right\rangle
$$

In the stochastic estimator technique, one uses (complex) random source vectors with the property

$$
\lim _{N_{E} \rightarrow \infty} \frac{1}{N_{E}} \sum_{E=1}^{N_{E}} \eta_{i}^{*}(E, C) \eta_{j}(E, C)=\delta_{i, j}
$$

and computes the quantity $\eta^{\dagger}(E, C) M^{-1}(C) \eta(E, C)$ repeatedly $\left(N_{E}\right.$ times) on each configuration.

It appears natural to choose $\eta(E, C)$ according to a Gaussian distribution (SETG) [6]. A decomposition similar to eq.(3) then yields

$$
\begin{aligned}
& \frac{1}{N_{C}} \sum_{C=1}^{N_{C}} \frac{1}{N_{E}} \sum_{E=1}^{N_{E}} \eta^{\dagger}(E, C) M^{-1}(C) \eta(E, C) P(C)= \\
& \quad \frac{1}{N_{C}} \sum_{C=1}^{N_{C}}\left\{\left[\sum_{i} M_{i, i}^{-1}(C)+\bar{T}_{o n}\left(N_{E}, C\right)+\bar{T}_{o f f}\left(N_{E}, C\right)\right] P(C)\right\},
\end{aligned}
$$

where

$$
\begin{aligned}
\bar{T}_{o n}\left(N_{E}, C\right) & =\frac{1}{N_{E}} \sum_{E=1}^{N_{E}} \sum_{i}\left[\eta_{i}^{*}(C, E) \eta_{i}(C, E)-1\right] M_{i, i}^{-1}(C) \\
\bar{T}_{o f f}\left(N_{E}, C\right) & \left.=\frac{1}{N_{E}} \sum_{E=1}^{N_{E}} \sum_{i \neq j} \eta_{i}^{*}(C, E) \eta_{j}(C, E)\right) M_{i, j}^{-1}(C) .
\end{aligned}
$$


Unfortunately the term $\bar{T}_{o n}\left(N_{E}, C\right)$ on the right hand side of eq.(6) introduces a gauge invariant bias, whose suppression definitely requires the number of estimates per configuration to be in the asymptotic regime, where eq.

This dangerous bias is removed from the beginning, if one samples the components of the random vector $\eta(E, C)$ according to a $Z_{2}$ distribution. In this case, the relation $\eta_{i}^{*}(E, C) \eta_{i}(E, C)=1$ holds for any single estimate $E$, and $T_{o n}$ vanishes on each gauge configuration.

For the remaining nonlocal bias, $\bar{T}_{\text {off }}\left(N_{E}, C\right)$, SETZ combines two additive suppression mechanisms: (a) the gauge fluctuations cancel them as non gauge invariant objects on a sufficiently large sample of gauge fields, even for $N_{E}=1$; (b) the $Z_{2}$-noise kills them in the large $N_{E}$ limit, even on a single gauge configuration.

In order to understand the efficiency of the competing methods for rendering good signals in more detail, we consider the variance $\sigma^{2}$ of $\left\langle P \operatorname{Tr}\left(M^{-1}\right)\right\rangle$ in each case. Asymptotically one finds

$$
\sigma^{2}=\sigma_{\text {gauge }}^{2}+\left\{\begin{array}{ll}
\frac{1}{N_{E}}\left\langle\sigma_{o f f}^{2} P^{2}\right\rangle & \text { for SETZ } \\
\frac{1}{N_{E}}\left\langle\left(\sigma_{o n}^{2}+\sigma_{o f f}^{2}\right) P^{2}\right\rangle & \text { for SETG } \\
\left\langle\left(\sum_{i \neq j} M_{i, j}^{-1}\right)^{2} P^{2}\right\rangle & \text { for VST }
\end{array}\right\}+2 \mathrm{COV}
$$

$\sigma_{\text {gauge }}^{2}$ is the variance calculated with the exact value of $\operatorname{Tr} M^{-1}$ on each configuration, and $\sigma_{o f f}^{2}$ and $\sigma_{o n}^{2}$ are the variances due to the distribution of $T_{o f f}$ and $T_{o n}$ within the process of stochastic estimation. The cornered brackets stand for the average over gauge configurations. The abbreviation COV in eq.8 reads in its full length

$$
C O V=\operatorname{cov}\left\{\begin{array}{ll}
\left(P \operatorname{Tr}\left(M^{-1}\right), P \bar{T}_{o f f}\right) & \text { for SETZ } \\
\left(P \operatorname{Tr}\left(M^{-1}\right), P\left(\bar{T}_{o n}+\bar{T}_{o f f}\right)\right) & \text { for SETG } \\
\left(P \operatorname{Tr}\left(M^{-1}\right), P \sum_{i \neq j} M_{i, j}^{-1}\right) & \text { for VST }
\end{array} .\right.
$$

These formulae show how the signals will be blurred by the fluctuations of the estimates $\operatorname{Tr}\left(M^{-1}(C)\right)$. Note that the terms in the large curly brackets of eq.8 are all gauge invariant. Hence they survive even in the limit of an infinite sample of gauge configurations unless suppressed otherwise : there is a way to fight them within SETZ and SETG according to a $1 / N_{E}$ suppression?, but there is no way to influence them at all within VST.

So far the discussion is qualitative only, as we do not know the relative size of these additional terms in eq. 8. Furthermore, the calculation of $\sigma_{N}$ requires the investigation of the ratio $\left\langle P(0 \rightarrow t) \operatorname{Tr}\left(M^{-1}\right)\right\rangle /\langle P(0 \rightarrow t)\rangle$ rather than $\left\langle P(0 \rightarrow t) \operatorname{Tr}\left(M^{-1}\right)\right\rangle$ itself. This

\footnotetext{
${ }^{1} \mathrm{~A}$ similar analysis has been carried out in [10 for a modified version of VST, applied to the calculation of the $\pi \pi$ scattering length.

${ }^{2}$ The terms depicted by COV behave like $\sqrt{1 / N_{E}}$ for SETZ and SETG. In case of SETG COV contains a gauge invariant part due to $\bar{T}_{\text {on }}$, which can be removed only in the limit $N_{E} \rightarrow \infty$. All other terms in COV vanish for $N_{C} \rightarrow \infty$, as they are not gauge invariant.
} 
can entail cancellations due to additional correlations between numerator and denominator. Last not least we have to keep in mind that these considerations are only valid in the asymptotic limits of the gauge and estimator samples. In the next section we will therefore present a numerical study of the situation, under actual working conditions of full QCD.

\section{$3 \quad$ Numerical Results}

Our present analysis of $\sigma_{N}^{\text {disc }}$ is based on 157 configurations from our ongoing Hybrid Monte Carlo run 8, 9] with two degenerate flavours of dynamical Wilson fermions. Here we work with $\kappa_{\text {sea }}=.157$, which amounts to a ratio $M_{\pi} / M_{\rho}=0.76(1)$, and to a quark mass of $m_{q} \simeq 1.3 m_{s}$.

1. To set the stage, we consider first the implications of the stochastic estimator technique on the chiral condensate, $\chi=\operatorname{Tr}\left(M^{-1}\right) / 12 \mathrm{~V}$ and compare the performance of Gaussian and $Z_{2}$-noises in full QCD. In Fig. 1] we show - on a given configuration - the standard error resulting from the two methods as a function of the number of estimates, $N_{E}$. Clearly SETZ is superior (by about a factor two) in obtaining a good signal for

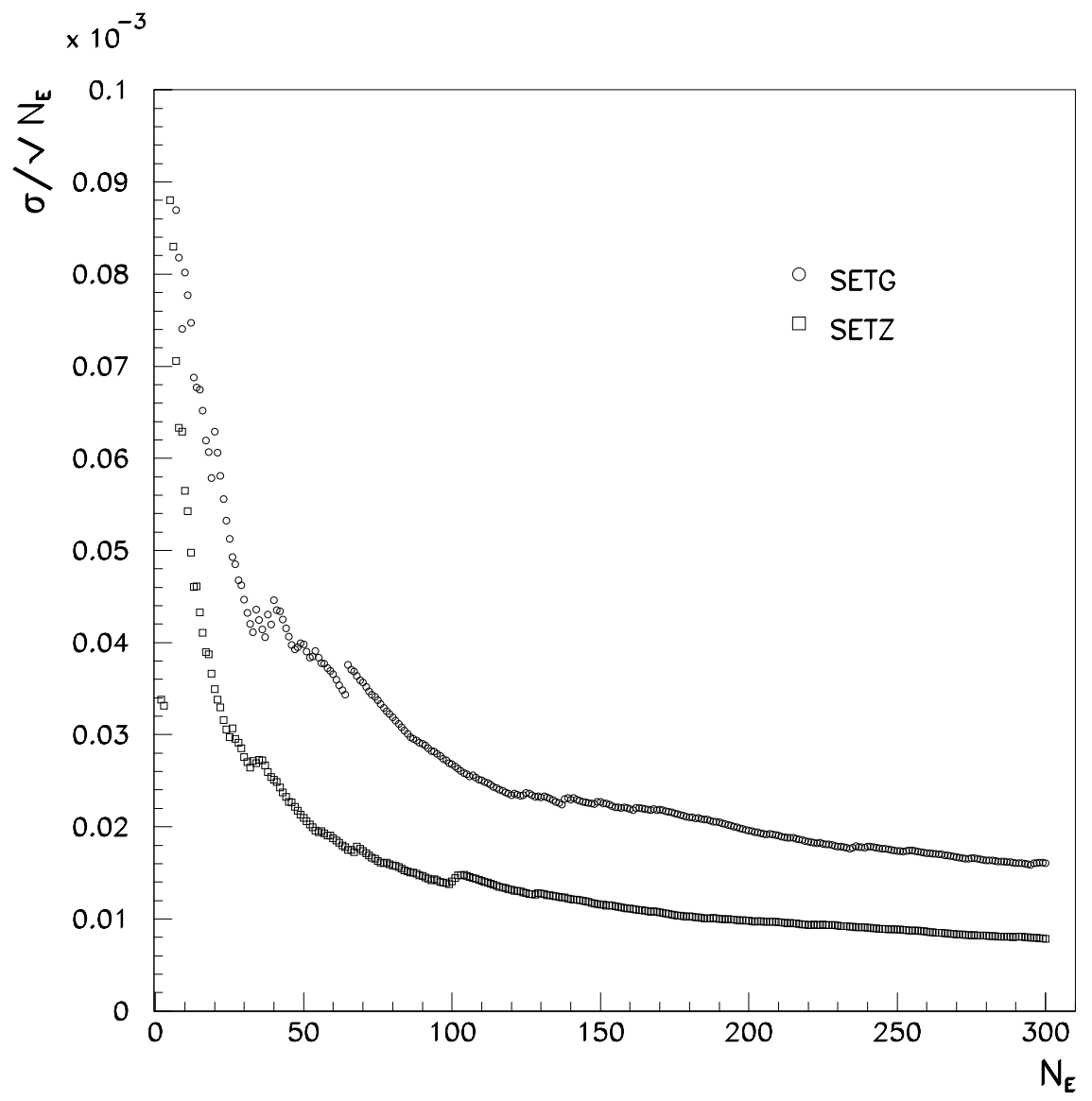

Figure 1: The standard error of $\chi$ for SETG and SETZ on a given configuration.

$\chi$; we note that this is similar to the related quenched situation[3]. For this reason we pursue SETZ in the following. 
2. An obvious way to economize is to properly adjust the accuracy in solving 11 eq.1. We therefore compute in the next step the chiral condensate in its dependence on the inversion accuracy $r=\|M X-h\| /\|X\|$. Fig.2 2 shows the convergence behaviour,

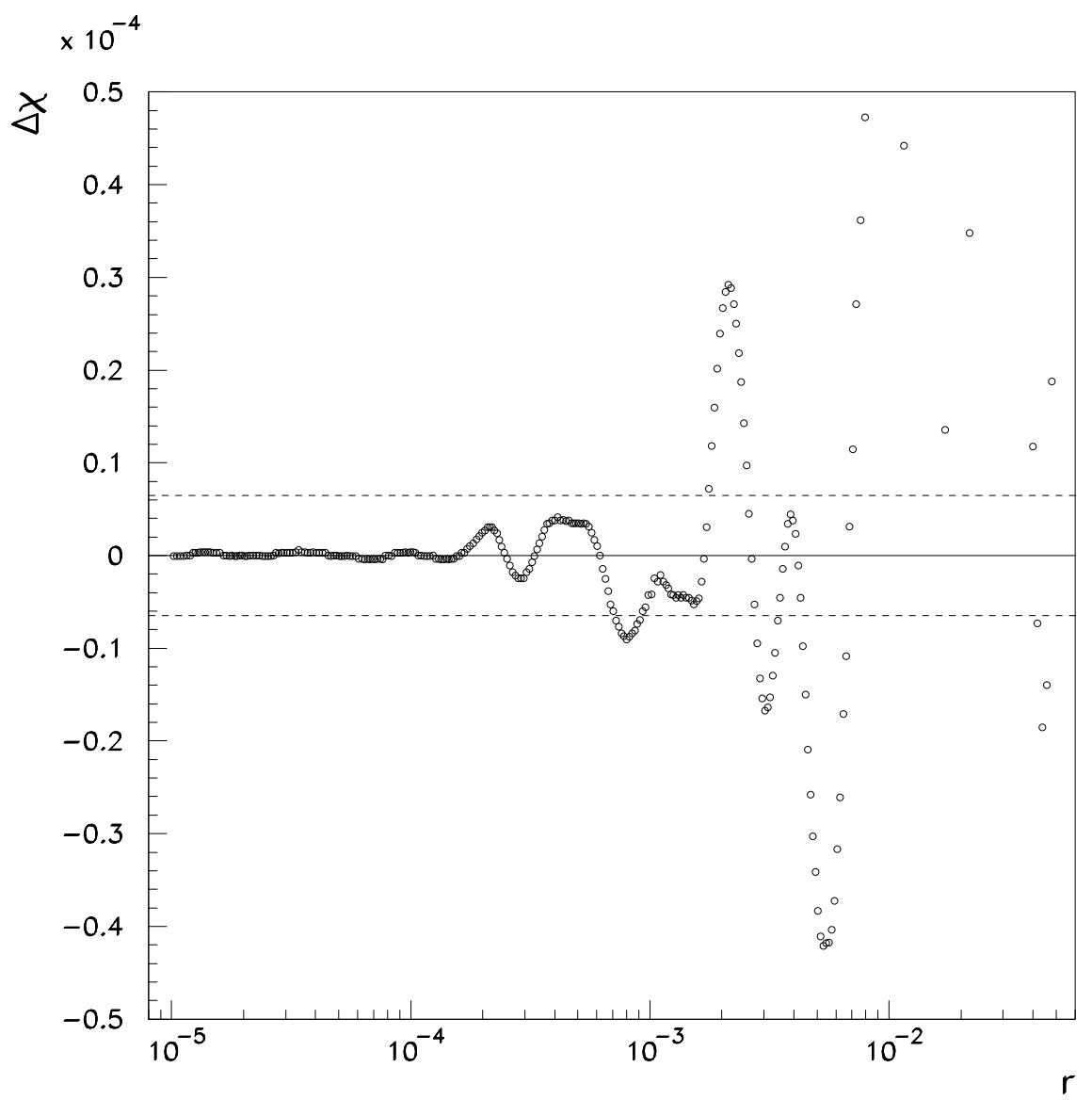

Figure 2: The difference $\Delta \chi(r)=\chi\left(r_{\max }=10^{-5}\right)-\chi(r)$ on a given configuration. The dashed horizontal lines indicate the one $\sigma$ margin from SETZ with $N_{E}=300$.

$\Delta \chi=\chi\left(10^{-5}\right)-\chi(r)$. The two horizontal lines in the plot refer to the $1 \sigma$ margin from the stochastic noise with $N_{E}=300$ estimators, on a single field configuration. It can be seen that below $r \simeq 10^{-4}, \Delta \chi$ is safely inside this margin. It is therefore sufficient to operate with the convergence condition $r \simeq 10^{-4}$.

3. The next question relates to the size of the sample of gauge configurations required to observe a signal of the scalar density matrix element $\langle P|\bar{q} q| P\rangle$. This quantity $\}^{3}$ is extracted from

$$
R(t)^{\text {disc }}=\frac{\left\langle P(0 \rightarrow t) \operatorname{Tr}\left(M^{-1}\right)\right\rangle}{\langle P(0 \rightarrow t)\rangle}-\left\langle\operatorname{Tr}\left(M^{-1}\right)\right\rangle \stackrel{t \text { large }}{\longrightarrow} \text { const }+t\langle P|\bar{q} q| P\rangle_{\text {disc }}^{\text {latt }} .
$$

To start we choose a large number of stochastic sources, $N_{E}=300$ as proposed for quenched simulations in Ref. 任, in order to avoid bias from the nonlocal pollutions to the trace. In Fig. 3 we show how the linear rise in $R(t)^{d i s c}$ evolves more and more clearly as the sampling is increased from 50 to 100 and 157 gauge configurations. The linear

\footnotetext{
${ }^{3}$ Throughout this work we applied gauge invariant gaussian smearing 12 with $N=50, \alpha=4$ to the Proton operator at the source.
} 

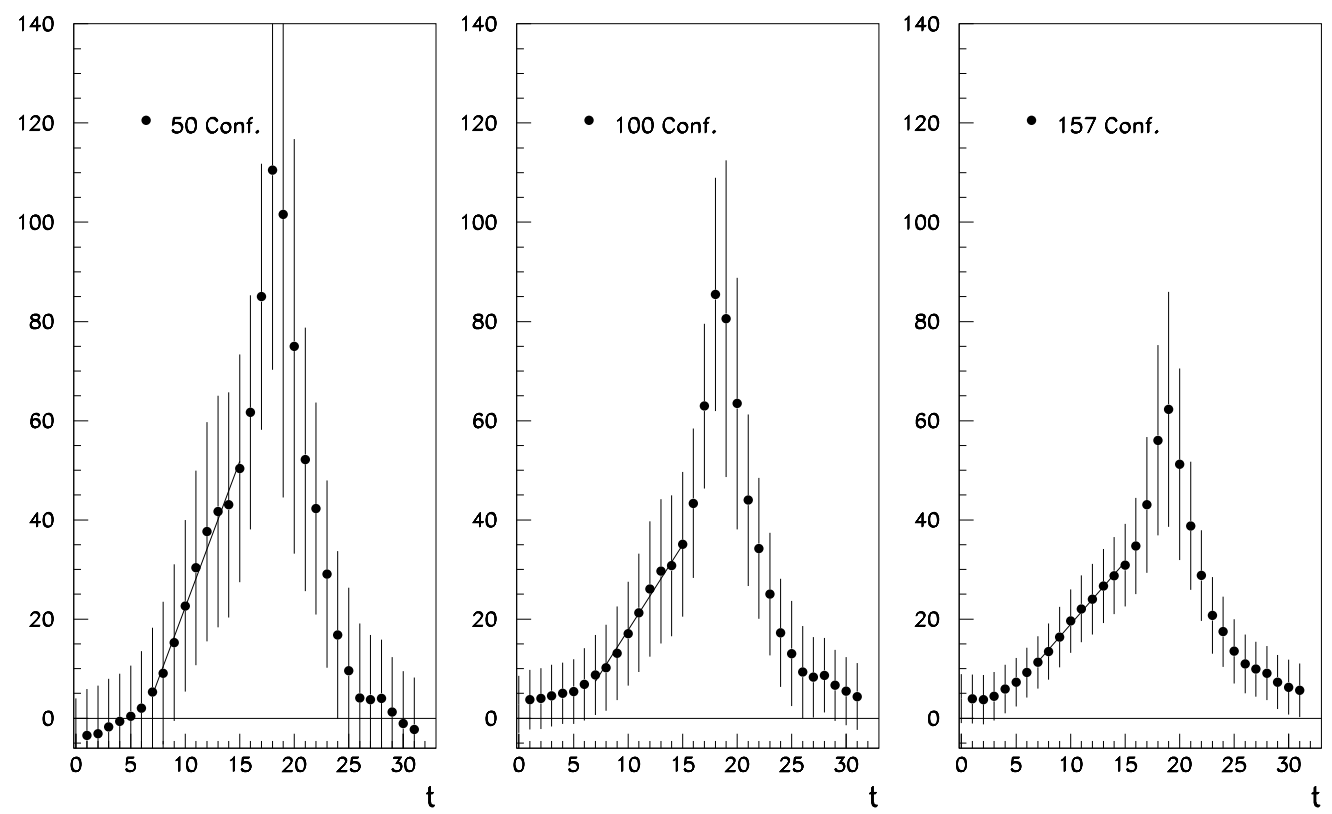

Figure 3: $R(t)^{\text {disc }}$ measured with SETZ $\left(N_{E}=300\right)$ on 50, 100 and 157 gauge field configurations. Linear fits were performed in the range $t=7$ to $t=15$.
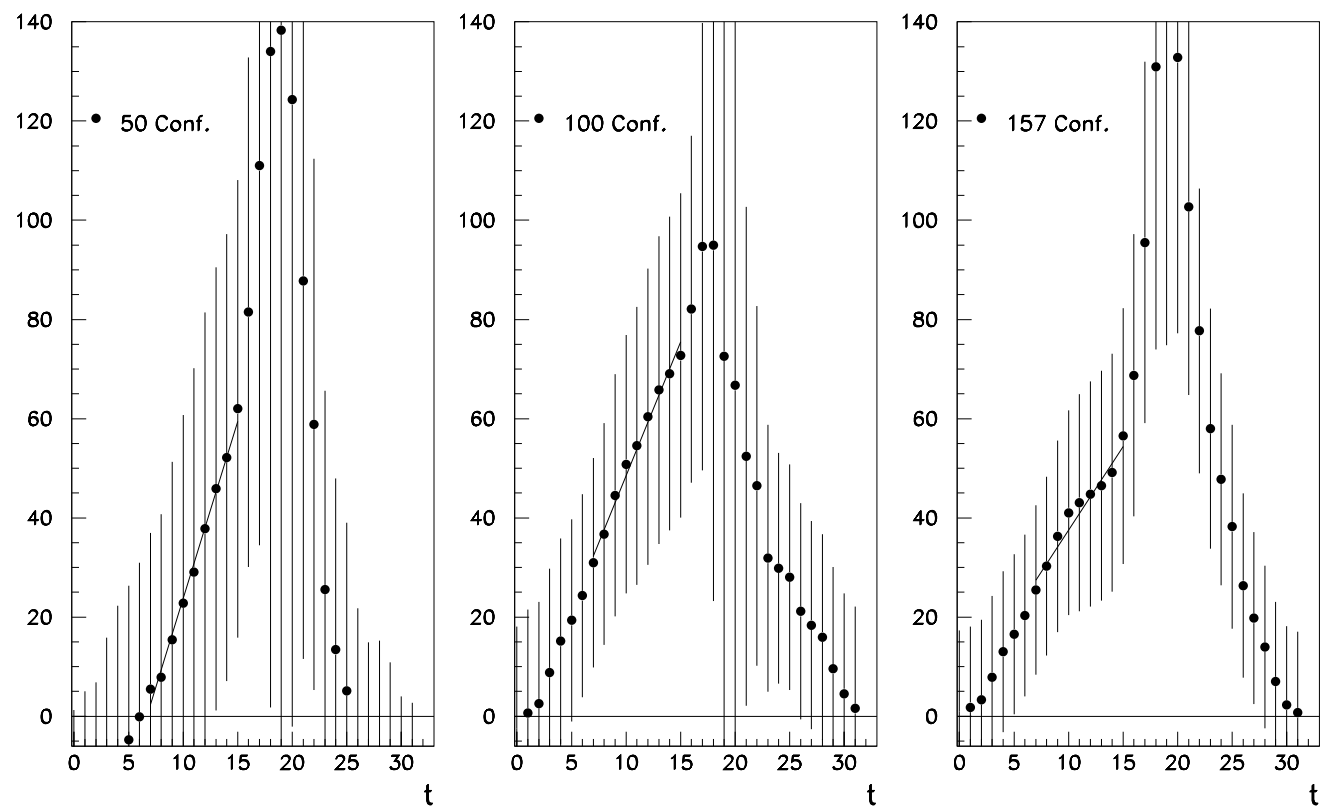

Figure 4: $R(t)^{\text {disc }}$ measured with VST on 50, 100 and 157 gauge configurations. Linear fits were performed in the range $t=7$ to $t=15$.

fits to this data yield the values 5.89(2.37), 3.44(1.45) and 2.51(0.77) for the slope.

We conclude that it appears mandatory to work on samples of at least 100 configurations. Under this condition we (a) retrieve a reasonable signal and (b) find the statistical 
errors on the data points to follow the expected behaviour $1 / \sqrt{N_{c}}$. For comparison we
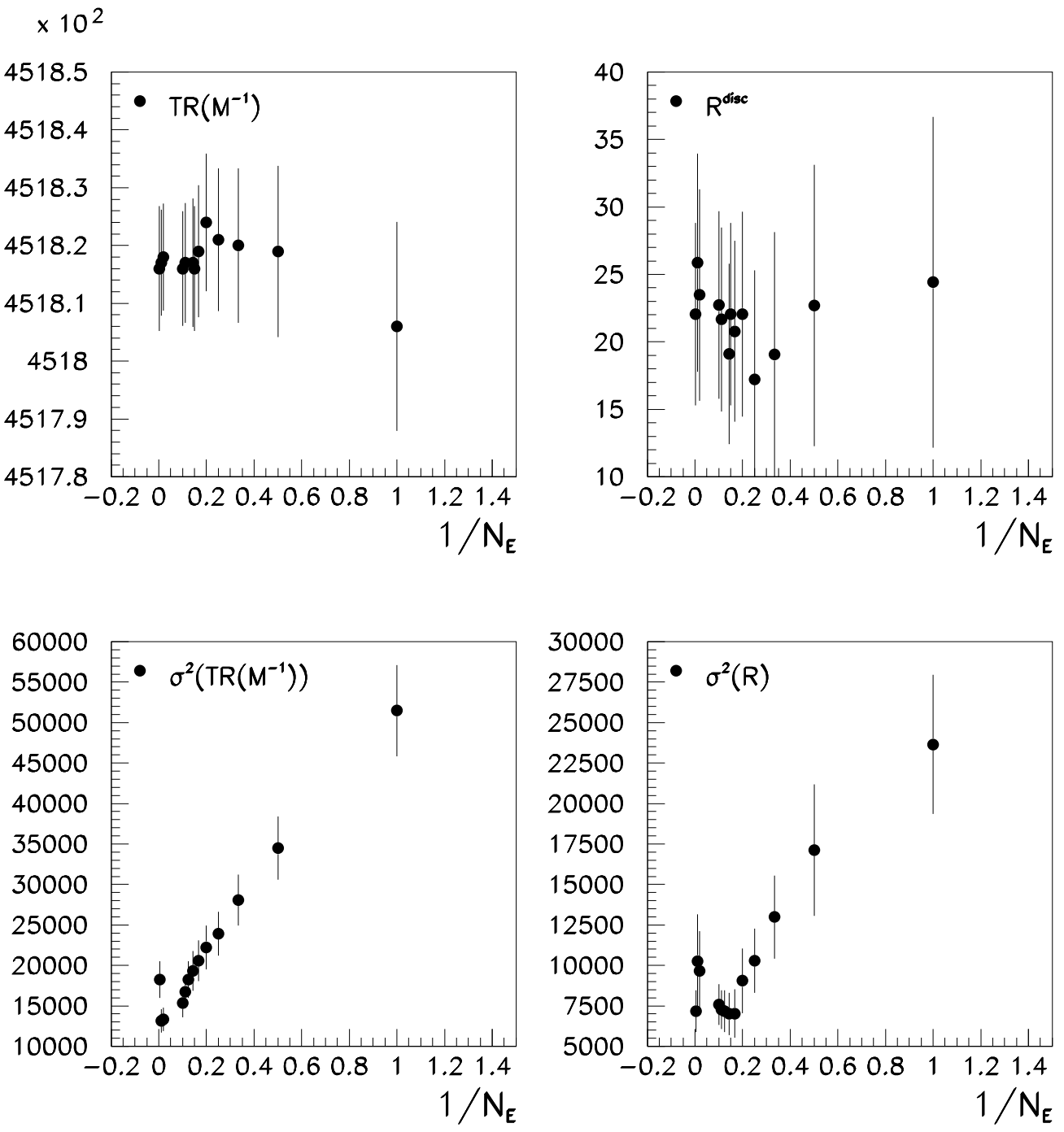

Figure 5: $\operatorname{Tr} M^{-1}, R^{\text {disc }}(t=11)$ and the corresponding variances as a function of $1 / N_{E}$. The data is taken from the full set of 157 gauge configurations.

show in Fig. $4(t)^{\text {disc }}$ calculated with VST on 50, 100 and 157 gauge configurations. Note that the signal to noise ratio is much worse in this case. For the slope we find 7.13(4.01), 5.40(2.8) and 3.50(2.20), consistent with SETZ, although with much larger statistical errors.

4. Given the signal on our present sample of 157 configurations we can now ask the question whether and how far we can actually relax in the number of stochastic estimates, $N_{E}$, without deteriorating its quality. The starting point is the observation that the fluctuations from SET add to the inherent fluctuations from the gauge field sample (see eq.8). For economics $N_{E}$ should be chosen just large enough to suppress this undesired effect. Fig. 5 shows the response of $\operatorname{Tr}^{-1}$ and of $R^{\text {disc }}(t=11)$ as well as the variances $\sigma^{2}\left(\operatorname{Tr} M^{-1}\right)$ and $\sigma^{2}\left(R^{\text {disc }}(t=11)\right)$ with respect to changes in $1 / N_{E}$. 

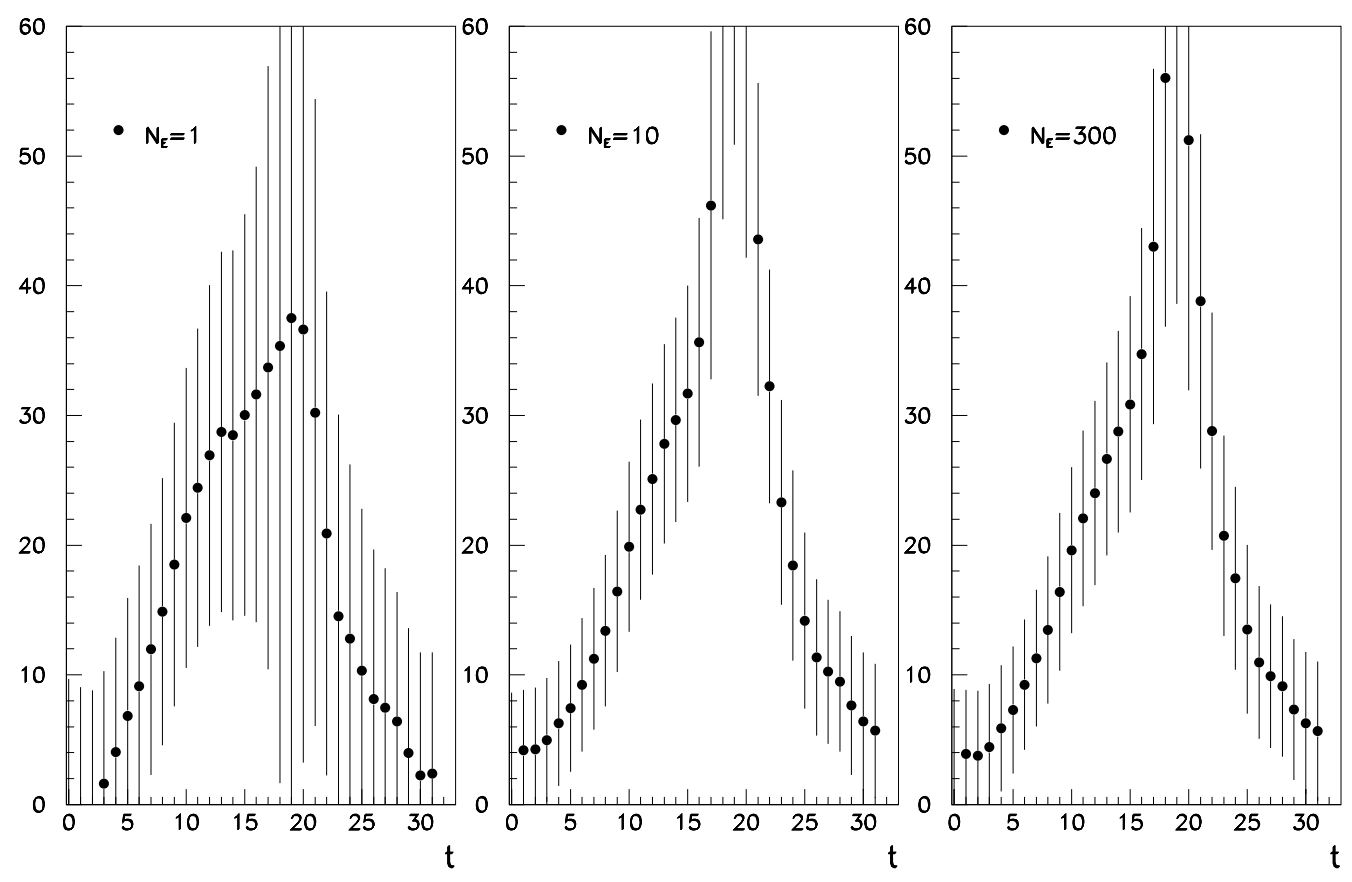

Figure 6: $R(t)^{\text {disc }}$ measured on 157 gauge configurations, with 1, 10 and 300 stochastic $Z_{2}$ estimates per configuration.

We find that the mean values are not affected by the variation of $N_{E}$ within the statistical accuracy. The variances however display a significant decrease over $1 \leq N_{E} \leq$ 10 , which ends in a somewhat noisy plateau. Obviously it does not pay to increase $N_{E}$ beyond a value of 10. For comparison we quote also the results obtained with VST on the identical sample of gauge configurations: $\operatorname{Tr} M^{-1}=451817, R^{\text {disc }}(t=11)=43.07$, $\sigma^{2}\left(\operatorname{Tr} M^{-1}\right)=121863(14582)$, and $\sigma^{2}\left(R^{\text {disc }}(t=11)\right)=75265(15073)$. Based on the variance we find SETZ to outperform VST substantially. In terms of statistics we recover a gain of a factor 2.5 to 3 from using SETZ instead of VST, in the present application. The full $t$-dependence of $R^{d i s c}(t)$ is displayed in Fig.6, showing again that 10 estimates within SETZ are sufficient to produce a reasonable signal on our sample of 157 configurations, while the signal is nil with one estimate only. On the other hand 300 estimates are definitely (and fortunately!) unnecessary.

\section{Conclusion and Outlook}

We have presented a full QCD study on the stochastic estimator technique applied to the disconnected diagrams of the scalar density. We found that it is indeed possible to achieve clean signals from only $\mathcal{O}(10) Z_{2}$-noise sources with a weak convergence requirement, $r \simeq 10^{-4}$ on the iterative solver to $\operatorname{Tr} M^{-1}$. This saves a factor $30 \mathrm{in}$ computer time compared to previous applications of this technique in the quenched applications.

With as few as 157 dynamical field configurations we obtain a reliable signal on $<P|\bar{q} q| P>_{\text {disc. }}$. This opens the door to perform a detailed analysis of the $\pi$-N $\sigma$ term 
as well as the axial vector matrix elements in the proper setting, i.e. without recourse to the quenched approximation. Work along this line is in progress.

It is obvious that in full QCD simulations the use of appropriately optimized $Z_{2^{-}}$ noise techniques will be of utmost importance when it comes to the estimate of more complex quantitites in flavour-singlet channels, where the underlying correlators contain two disconnected fermion loops.

Exploratory quenched applications of VST to compute the $\eta^{\prime}$-mass and the annihilation diagrams in $\pi \pi$-scattering in the isospin zero channel at threshold have attained good signals [13, 14]. This makes us hope that medium size sampling will lead in full QCD to reliable signals as well, once optimized estimator techniques will be applied to grasp the loops.

Acknowledgements. We are grateful to DESY, DFG and KFA for substantial computer time on their QH2 Quadrics systems at DESY/Zeuthen, University of Bielefeld and on the Q4 and CRAY T90 systems at ZAM/KFA. Thanks to Hartmut Wittig, Markus Plagge and Norbert Attig for their kind support. This research has been supported by DFG (grants Schi 257/1-4 and Schi 257/3-3) and by EU contracts SC1*-CT91-0642 and CHRX-CT92-0051.

\section{References}

[1] R. Altmeyer, M. Göckeler, R. Horseley, E. Laermann, and G. Schierholz, Nucl. Phys. B (Proc.Suppl.) 34(1994)376.

[2] J.E. Mandula, M.C. Ogilvie, Phys. Lett.B312(1993)327.

[3] S.J. Dong and K.F. Liu, Nucl.Phys. B (Proc.Suppl.) 26(1992)353; Phys. Lett.B328(1994)130.

[4] S.J. Dong and K.F. Liu, Nucl.Phys. B (Proc.Suppl.) 26(1993)487; K.F. Liu, S.J. Dong, T. Draper and W. Wilcox, Phys. Rev. Lett.74(1995)2172; S.J. Dong, J.F. Lagaë, and K.F. Liu, Phys. Rev. Lett.75(1995)2096.

[5] Y. Kuramashi, M. Fukugita, H. Mino, M. Okawa, and A. Ukawa, Phys. Rev. Lett.75(1995)2092; Phys. Rev. D 51 (1995)5319.

[6] Bitar et al., Nucl. Phys. B313(1989)348.

[7] for a review, see M. Okawa, Nucl. Phys.B (Proc.Suppl.) 47(1996)160.

[8] SESAM Collaboration: U. Glässner, S. Güsken, H. Hoeber, Th. Lippert, X. Luo, G. Ritzenhöfer, K. Schilling, and G. Siegert, Nucl. Phys.B (Proc.Suppl.) 47(1996)386.

[9] SESAM Collaboration: N. Eicker, U. Glässner, S. Güsken, H. Hoeber, Th. Lippert, X. Luo, G. Ritzenhöfer, K. Schilling, G. Siegert, and J. Viehoff, in preparation.

[10] M. Fukugita, Y. Kuramashi, M. Okawa, H. Mino and A. Ukawa, Phys. Rev. D 52 (1995)3003. 
[11] A. Frommer, V. Hannemann, B. Nöckel, T. Lippert and K. Schilling, Int. J. Mod. Phys. C5 (1994) 1073.

[12] S. Güsken, U. Löw, K.H. Mütter, R. Sommer, A. Patel and K. Schilling, Nucl. Phys. B (Proc.Suppl.) 17 (1990)361.

[13] Y. Kuramashi, M. Fukugita, H. Mino, M. Okawa, and A. Ukawa, Phys. Rev. Lett.72(1994)3448.

[14] Y. Kuramashi, M. Fukugita, H. Mino, M. Okawa, and A. Ukawa, Phys. Rev. Lett.75(1993)2387. 